\title{
Electronic and Geometric Structure of Transition-Metal Nanoclusters
}

\section{RECEIVED \\ AUG 161996 \\ OSTI}

\author{
D. R. Jennison, P. A. Schultz, M. P. Sears, T. Klitsner
}

\section{Prepared by}

Sandia National Laboratories

Albuquerque, New Mexico 87185 and Livermore, California 94550

for the United States Department of Energy

under Contract DE-AC04-94AL85000

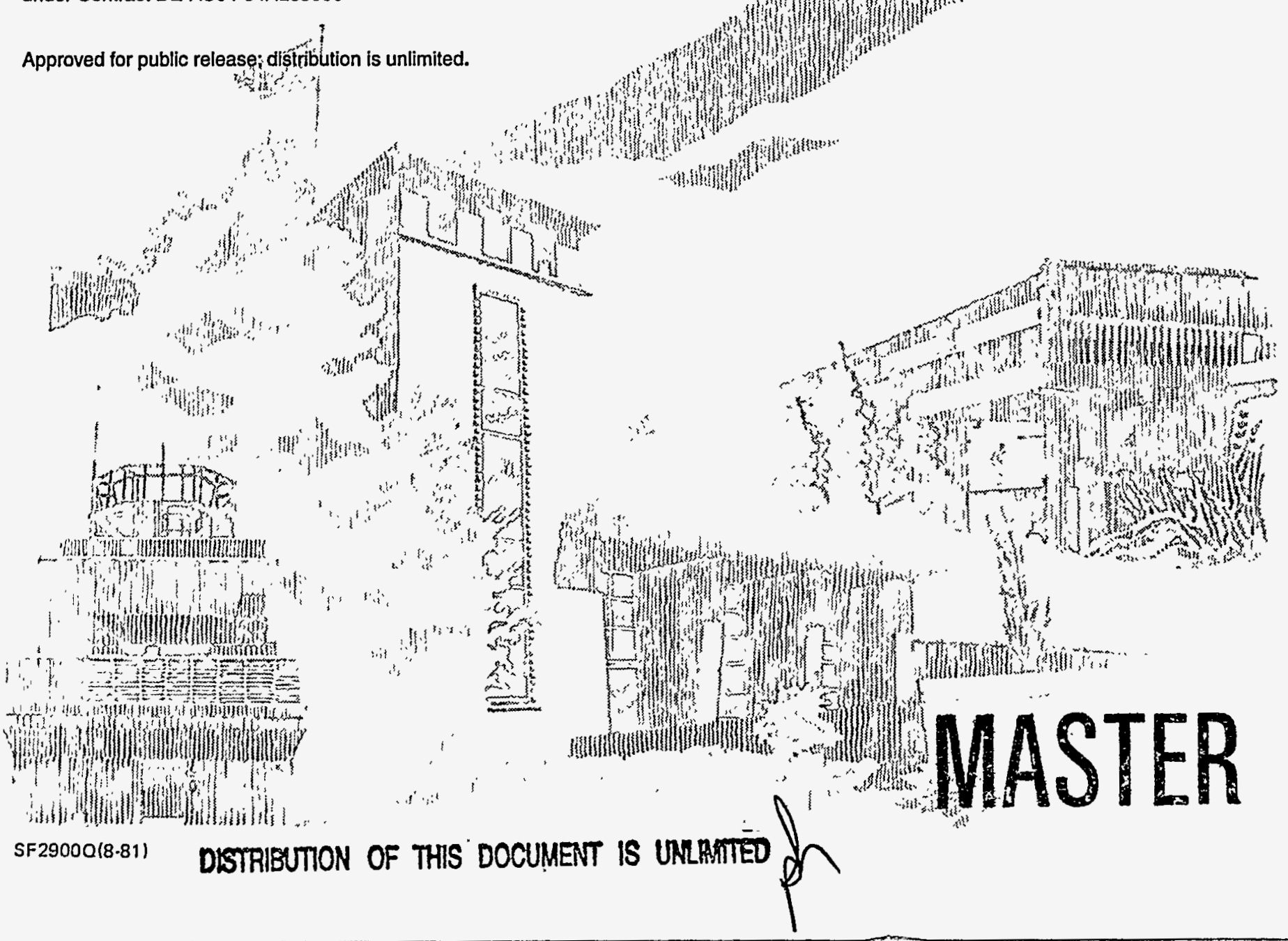


Issued by Sandia National Laboratories, operated for the United States Department of Energy by Sandia Corporation.

NOTICE: This report was prepared as an account of work sponsored by an agency of the United States Government. Neither the United States Government nor any agency thereof, nor any of their employees, nor any of their contractors, subcontractors, or their employees, makes any warranty, express or implied, or assumes any legal liability or responsibility for the accuracy, completeness, or usefulness of any information, apparatus, product, or process disclosed, or represents that its use would not infringe privately owned rights. Reference herein to any specific commercial product, process, or service by trade name, trademark, manufacturer, or otherwise, does not necessarily constitute or imply its endorsement, recommendation, or favoring by the United States Government, any agency thereof or any of their contractors or subcontractors. The views and opinions expressed herein do not necessarily state or reflect those of the United States Government, any agency thereof or any of their contractors.

Printed in the United States of America. This report has been reproduced directly from the best available copy.

Available to DOE and DOE contractors from

Office of Scientific and Technical Information

PO Box 62

Oak Ridge, TN 37831

Prices available from (615) 576-8401, FTS 626-8401

Available to the public from

National Technical Information Service

US Department of Commerce

5285 Port Royal Rd

Springfield, VA 22161

NTIS price codes

Printed copy: A03

Microfiche copy: A01 


\section{DISCLAIMER}

Portions of this document may be illegible in electronic image products. Images are produced from the best available original document. 
, 


\section{DISCLAIMER}

This report was prepared as an account of work sponsored by an agency of the United States Government. Neither the United States Government nor any agency thereof, nor any of their employees, makes any warranty, express or implied, or assumes any legal liability or responsibility for the accuracy, completeness, or usefulness of any information, apparatus, product, or process disclosed, or represents that its use would not infringe privately owned rights. Reference herein to any specific commercial product, process, or service by trade name, trademark, manufacturer, or otherwise does not necessarily constitute or imply its endorsement, recommendation, or favoring by the United States Government or any agency thereof. The views and opinions of authors expressed herein do not necessarily state or reflect those of the United States Government or any agency thereof. 

SAND 96-1745

Distribution

Unlimited Release

Printed August 1996

\title{
ELECTRONIC AND GEOMETRIC STRUCTURE OF TRANSITION- METAL NANOCLUSTERS
}

\author{
D. R. Jennison ${ }^{a}$, P. A. Schultz ${ }^{b}$, M. P. Sears ${ }^{b}$, and T. Klitsner ${ }^{c *}$ \\ a) Surface and Interface Sciences Department 1114 \\ b) Parallel Computational Sciences Department 1421 \\ c) Fuel Science Department 6211 \\ Sandia National Laboratories, Albuquerque, NM 87185-1413
}

\begin{abstract}
A massively-parallel $a b$ initio computer code, which uses Gaussian bases, pseudopotentials, and the local density approximation, permits the study of transition-metal systems with literally hundreds of atoms. We present total energies and relaxed geometries for $\mathrm{Ru}, \mathrm{Pd}$, and $\mathrm{Ag}$ clusters with $\mathrm{N}=55,135$, and 140 atoms; we also used the DMOL code to study 13-atom Pd and Cu clusters, with and without hydrogen. The $\mathrm{N}=55$ and 135 clusters were chosen because of simultaneous cubo-octahedral (fcc) and icosahedral (icos) sub-shell closings, and we find icos geometries are preferred. Remarkably large compressions of the central atoms are observed for the icos structures (up to $6 \%$ compared with bulk interatomic spacings), while small core compressions ( 1\%) are found for the fcc geometry. In contrast, large surface compressive relaxations are found for the fcc clusters ( $\sim 2-3 \%$ in average nearest neighbor spacing), while the icos surface displays small compressions $(\sim 1 \%)$. Energy differences between icos and fcc are smallest for Pd, and for all systems the single-particle densities of states closely resembles bulk results. Calculations with $\mathrm{N}=$ 134 suggest slow changes in relative energy with $N$. Noting that the 135 -atom fcc has a much more open surface than the icos, we also compare $N=140$ icos and fcc, the latter forming an octahedron with close packed facets. These icos and fcc clusters have identical average coordinations and the octahedron is found to be preferred for $\mathrm{Ru}$ and $\mathrm{Pd}$ but not for $\mathrm{Ag}$. Finally, we compare Harris functional and LDA energy differences on the $\mathrm{N}=140$ clusters, and find fair agreement only for Ag.

* Present organization Dept. 4423.
\end{abstract}

FY 94 LDRD Final Report 


\section{Contents}

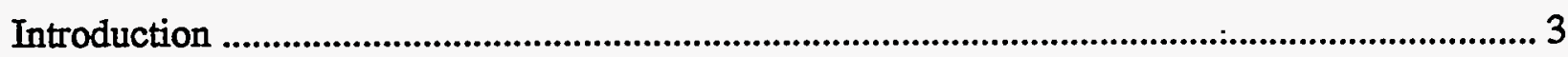

Studies of Large Ru, Pd, and Pt Nanoclusters Using the QUEST Code ........................................ 5

1. Computational Detail and Code Development .................................................................... 5

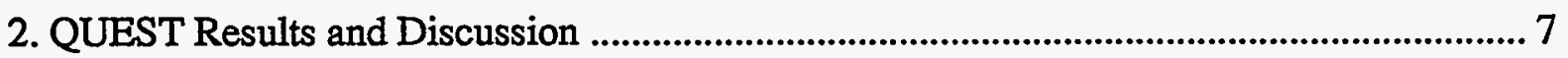

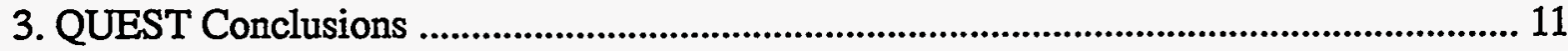

Studies of Small Pd and Cu Nanoclusters Using the DMOL Code ................................................ 12

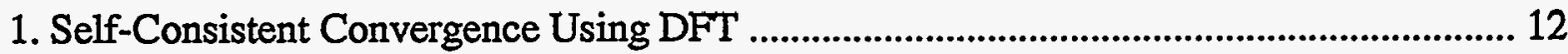

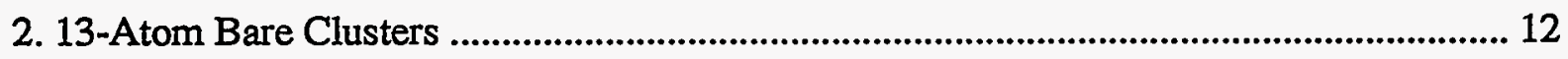

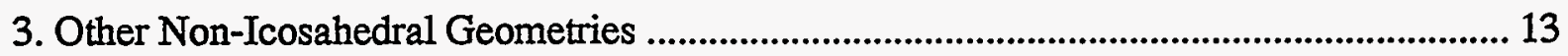

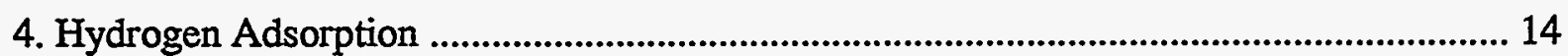

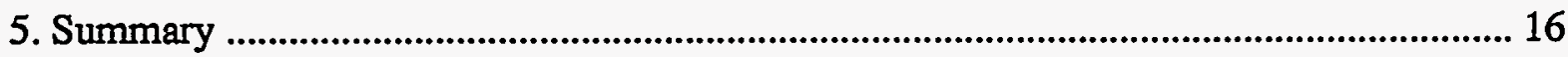

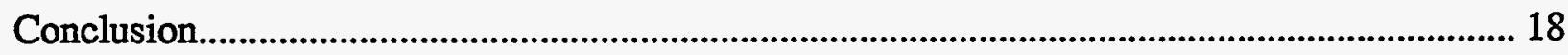

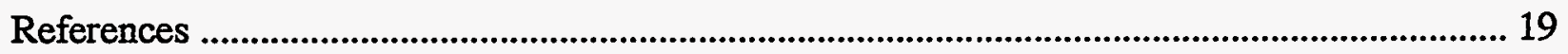

\section{APPENDIX -}

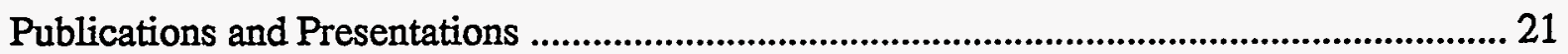

\section{Figures}

1. The 55-atom fec and icosahedral clusters ........................................................................... 22

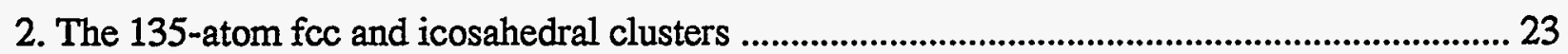

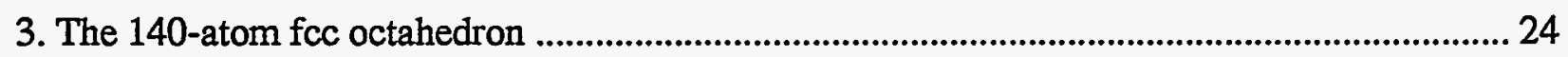

4. The DOS of the 135 atom icosahedral (solid) and fcc (dashed) Ru clusters ............................ 25

5. The DOS of the 135 atom icosahedral (solid) and fcc (dashed) Pd clusters ............................ 26

6. The DOS of the 135 atom icosahedral (solid) and fcc (dashed) Ag clusters ........................... 27

\section{Tables}

1. Basis set tests: "single vs. double zeta" (1d vs. 2d).................................................................... 28

2. Energy differences per atom of the fcc with respect to icosahedral structures (in $\mathrm{eV}$ ) ............. 28

3. Harris-functional total energy differences for $\mathrm{N}=140$ vs. full LDA....................................... 28

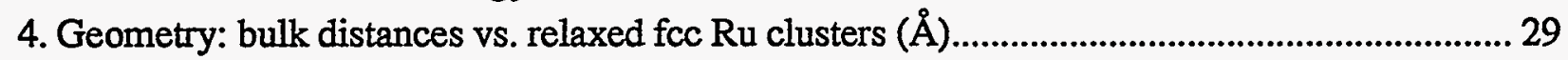

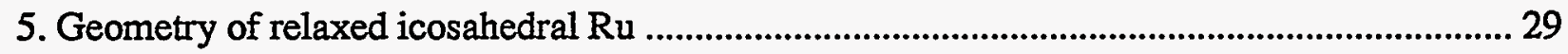

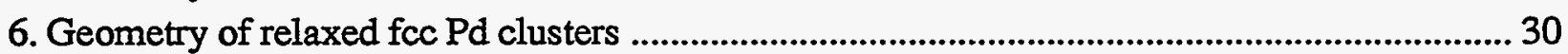

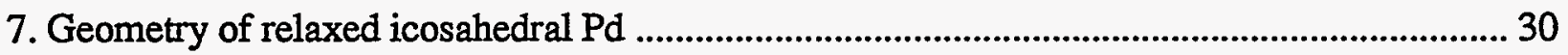

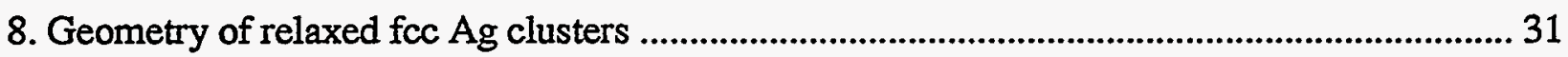

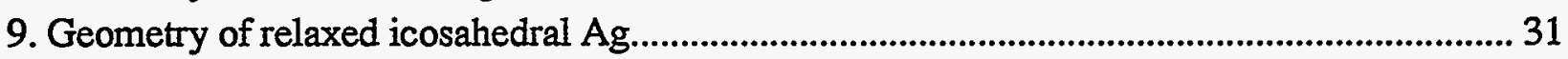

10. Geometry of the relaxed fcc octahedra................................................................................ 32

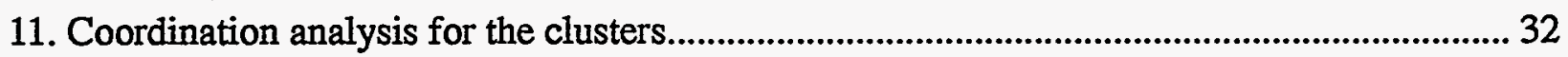




\section{Introduction}

Revolutionary advances in manufacture have made macroscopic quantities of nanoclusters cheaply available. Large quantities can be made by wet chemical [1], electrochemical [2], nucleated growth [3], and inverse micelle [4] techniques, spanning sizes from just a few to tens of thousands of atoms. In addition, with the inverse micelle technique, the mass of the clusters can be controlled to within 5-10\% and the resulting clusters are covered by surfactants which prevent clumping. Availability, and the intriguing potential to tailor these clusters for use in catalysis, have sparked a heightened interest in nanoclusters [5].

The geometries and other properties of "small" nanoclusters, of order 200 atoms or less, are largely unknown. However, interesting catalytic properties have been observed that cannot be explained by simple dispersion [1, 2, 6], and are possibly due to fundamental differences in structure from larger clusters. In this paper we examine the structures of metallic nanoclusters using first principles local density functional calculations and report on the relative stabilities of icosahedral (icos) vs. cubo-octahedral (fcc) geometries of clusters composed of the transition metals $\mathrm{Ru}, \mathrm{Pd}$, and of the noble metal Ag.

Microscopic gas-phase quantities of small clusters can be made using laser ablation, producing metal vapor that condenses in helium flow tubes [7], and permitting the clusters to be studied in vacuum. In the last several years, a few groups have developed the ability to perform photoemission experiments [8] or to place the clusters on a support for microscopy [9]. While, with some notable exceptions [10], small semiconductor nanoclusters seem likely to reflect the directional or ionic bonding present in bulk materials, little is known concerning the structural details of transition metal clusters [1]. The most information comes from the Argonne group, who has studied adsorption properties by injecting molecules in flow tubes and counting, by mass spectroscopy, the number that stick as a function of nanocluster metal mass and temperature [11]. For different adsorbates, "rules" have been established concerning coverage and site preference, and cluster structures have been inferred. Generally, icosahedral-based structures are observed in clusters to over 100 atoms, with polyicosahedral forms where atom numbers are well-between the 
major icosahedral shell closings of 13,55 , and 147 [12]. Naturally, a multitude of nearly degenerate isomers is possible. It is desirable to know the relative stability and geometries of icosahedralbased (icos) vs. bulk terminated cubo-octahedral (or face-centered cubic - $\mathrm{fcc}$ ) structures.

Icosahedral geometry near major shell closings maximizes the coordination of surface atoms while minimizing their number (see Figs. 1-2); however, the center of the cluster must be compressed if surface atoms are to have near typical interatomic distances. In contrast, while fcc structures do not require core compression for the surface atoms to be spaced "properly" (although a small amount is expected from the "surface tension"), a competition exists between minimizing surface area and maximizing the average surface atom coordination. Note that the surface-area-minimized fcc structures seen in Figs. 1-2 produce a mix of close-packed and more open microfacets, the latter nominally raising the surface energy compared with comparable icos forms. On the other hand, maximizing surface coordination in fcc structures increases the surfaceto-bulk atom ratio, while lowering the surface energy at the expense of the bulk energy (see Fig. 3). With increasing cluster size, at some point the cost of bulk compression is too high to sustain the icos structure, and we expect bulk-based structures to take over; faceting in then determined by the relative surface energies. It is unknown, however, where this transition occurs. It is also unknown which structures are favored for specific materials in view of the above competing factors.

Many-body (i.e., three center and higher) contributions to the total energy are well-known to be important in metals and preclude prediction based on simple theoretical models, such as tightbinding approaches. Embedded-atom models can predict surface energies poorly and incorrectly produce surface relaxations in several cases, such as $\mathrm{Pd}$ and $\mathrm{Pt}$ (111) [13]. Thus more sophisticated theory is indicated, which can also serve to test the more approximate methods.

In this paper, we report local density functional $a b$ initio calculations on the relative stability and geometries of icos vs. fcc structures of relatively large clusters of the transition metals Ru and $\mathrm{Pd}$ and of the noble metal Ag. Because of simultaneous sub-shell closings, we first study clusters of 55- and 135- atoms, as these avoid the more complex structures which are likely to occur well- 
between shell closings. We chose these materials to include in our study significant differences in cohesive energies and metallic bonding. We also report on 134 atom clusters, in order to see the magnitude of the relative total energy change upon deviating from subshell closing, and on 140atom icosahedra vs. the fcc octahedron (Fig. 3), in order investigate the competition between surface and bulk energy described above. Finally, we report studies of 13 atom clusters $\mathrm{Pd}$ and $\mathrm{Cu}$ clusters, with and without hydrogen.

The 55- and 135-atom clusters were constructed by placing successive shells of either fec or icos symmetry around a central atom. The 134 atom clusters were constructed by removing one atom of lowest coordination from the 135 atom clusters. The 140-atom octahedron was constructed by placing successive fcc shells about the $(1 / 2,1 / 2,1 / 2)$ lattice position (the octahedral void). The 140-atom icosahedron was constructed by adding 5 atoms at sites of maximal coordination (those with five-fold symmetry) on the 135 atom icosahedron. All geometries were then fully relaxed using the computed LDA forces.

\section{Studies of Large Ru, Pd, and Pt Nanoclusters Using the QUEST Code}

\section{Computational Details and Code Development}

The large-scale calculations required for this study were performed using the new Gaussianbased density-functional code QUEST (QUantum Electronic STructure) [14]. QUEST was specially designed and expressly written for massively parallel (MP) distributed-memory computer architectures. The Gaussian atom-centered orbital scheme is key to the implementation of a very efficient good-scaling method that readily incorporates the d-orbitals necessary to study transition metals. With $\mathrm{N}$ being the number of atoms, over the range of cluster sizes considered in this paper, the computational time scales as $\mathrm{N}^{1.3}$. This scaling, coupled with MP computing power, enables studies of clusters and periodic systems with hundreds of atoms.

The nanocluster work was the driving force behind a significant portion of QUEST development, especially that targeted toward metals. Under the LDRD, the atom potential/basis-set library used by QUEST was greatly expanded and tested. For example, the late $4 d$ transition metal 
bases were built, and benchmarked other calculations, and against bulk properties. In addition, much of the work involved comparing cluster properties with bulk materials, and the LDRD supported work to complete and validate the incorporation of periodic boundary conditions in the code, including code taking advantage of symmetry that enables use of a sampling of Bloch vectors reduced to the irreducible Brillouin zone.

During the course of the LDRD, calculated forces were finally integrated into QUEST, and the nano-cluster work represented the first concrete application of the use of forces for problems beyond test cases. For analysis of the results of the calculations, some post-processing capabilities were added. In order to understand some of the results, it proves necessary to render the electronic charge densities of the systems studied, and toward this end, special I/O was added to QUEST to provide the necessary input to a new code written to visualize charge densities, and differences between charge densities.

The local density approximation (LDA) [15], to density functional theory [16], is known to yield excellent geometries and relative energies for metal systems, and has achieved considerable success in studies of complex metal surfaces and metal adatom motion on surfaces [17]. Gradient corrections [18] have proven not to improve on standard LDA for metal structure. In our calculations, we use the Perdew/Zunger LDA parameterization [19] of the Ceperley/Alder electron gas results [20], and employ the generalized norm-conserving pseudopotentials of Hamann [21] to remove the chemically uninteresting core electrons from the problem. We neglect spin-polarization, for while magnetism has been observed for $\mathrm{Rh}$ nanoclusters [22], it has not been observed for the materials considered here.

The atomic basis sets used for the transition metal atom [23] consisted of three Gaussian functions contracted to give to s-functions, two to give a single p-function, and five to give one d-function ( $3 \mathrm{~s} 2 \mathrm{p} 5 \mathrm{~d} / 2 \mathrm{~s} 1 \mathrm{p} 1 \mathrm{~d})$. A “double-zeta" d-function ( $3 \mathrm{~s} 2 \mathrm{p} 5 \mathrm{~d} / 2 \mathrm{~s} 1 \mathrm{p} 2 \mathrm{~d}$ ) giving a second radial degree of freedom for the d-orbitals proved unnecessary, as using single-zeta bases yields relaxed geometries and relative energies in good agreement for a variety of test case. For example, Table 1 lists bulk properties computed with these bases. The relative energetics of relaxed icos and fcc clusters 
changed only negligibly upon promoting the basis. In order to achieve near-linear scaling, the code employs an algorithm due to Feibelman [24] that entails dividing the evaluation of matrix elements of the Hamiltonian into a local atom-centered component containing all the rapidly varying character, and a slowly varying component evaluated on a regular mesh. A mesh spacing of 0.35 Bohr proved sufficient to converge the numerical quadratures.

To achieve a self-consistency of $10^{-6}$ Rydberg $(1 \mathrm{Ry}=13.6 \mathrm{eV})$ in total energy and to compute the forces required roughly two hours on 512 nodes of Sandia's Intel Paragon computer. A finite Fermi temperature $(0.1 \mathrm{eV})$ was required to achieve self-consistent convergence. Tests showed that this finite temperature did not significantly affect the relative energies or geometries, but was sufficient to prevent any Jahn-Teller distortion caused by degeneracies at the Fermi level. (We consider this distortion relatively unimportant in clusters of this size - the energetics are determined by Coulomb interactions which scale as $\sim 1 / \mathrm{r}^{2}$, where $\mathrm{r}$ is the cluster radius compared with an atomic radius; in the present case, this amounts to $3-4 \%$ of atomic valence integrals, or a few $10^{-3} \mathrm{eV}$ per atom; cf. Table 2, below.)

The Pulay correction [25] required for a LCAO scheme were included in the computed forces. The clusters were relaxed without symmetry constraints through an iterative Broyden scheme developed by Johnson [26]. Six geometry steps typically sufficed to arrive at a "relaxed" structure, deemed achieved when forces on all atoms fell below $0.002 \mathrm{Ry} / \mathrm{Bohr}$.

\section{QUEST Results and Discussion}

The relative stabilities of relaxed 55- and 135-atom clusters are displayed in Table 2, presented on a per-atom basis to facilitate comparisons between results for different cluster sizes and for different elements. The icos geometries are more stable than the fcc geometries for all these clusters. In fact, contrary to expectation, the relative stability of the icos structure increases in going to the larger cluster for Ru. Furthermore, despite pronounced differences in metal-metal interactions, the results for $\mathrm{Ru}$ are more similar to $\mathrm{Ag}$, than either is to $\mathrm{Pd}$, looking at the magnitude of the relative stability of icos and fcc. This preference for icos does not appear to be markedly changed with small perturbations on the cluster. In the case of $\mathrm{Ag}$, the relative preference 
toward icos changes by only $0.002 \mathrm{eV} /$ atom to $0.027 \mathrm{eV} /$ atom in going from the 135 -atom cluster to a relaxed 134-atom cluster. In contrast, larger deviations from shell-closing can lead to very different results. Energy comparisons of icos and fcc geometries for 140-atom clusters, also listed in Table 2, reverse the order for $\mathrm{Ru}$ and Pd, with the fcc octahedron favored over the icos structure.

Relative to fully self-consistent LDA calculations, Harris functional [27] total energies are relatively simple to compute, and have been tried in molecular dynamics simulations of cluster structure [28]. However, to compute geometries using dynamical methods requires accuracy away from equilibrium as well as at a relaxed structure. To test the accuracy of the Harris functional for the materials studied here, we computed the Harris functional icos-fcc energy differences and compared them to the full LDA energy differences for our final relaxed structures as well as our initial guess geometries, "It. 0" (see Table 3). The results show the Harris functional does a poor job at computing the energy differences for Ru and Pd clusters, being in error by factors of 2-3. Given that the method approximates the total charge density as a superposition of spherical atomic densities, it is understandable that it does a much better job for $\mathrm{Ag}$, with errors in the $15 \%$ range. Apparently, the Harris functional is inadequate to deal with the angular character of the charge density in atoms such as Ru and Pd, with more localized binding resulting from open dshells.

In Tables 4-10, we compare the relaxed geometries of 55- and 135-atom fcc and icos clusters for $\mathrm{Ru}, \mathrm{Pd}$, and $\mathrm{Ag}$, and the 140-atom fcc octahedra. Listed are the number of atoms in each subshell about the central atom. For the fcc structures, the position vectors (in units of one-half the lattice constant), that under octahedral symmetry uniquely define each shell, are also listed, along with the shell radii that would result from using the bulk LDA interatomic spacings of an unrelaxed terminated-bulk cluster. Note that the nature of the relaxation in each of the clusters is the same regardless of atom, and bear out the qualitative predictions regarding the structures made in the Introduction. Rather large compressions about the central atom, up to 5-6\%, are observed for the icosahedral cluster, while surface relaxations are more modest $(\sim 1 \%$ decrease in average nn 
distance). In contrast, the fcc clusters have small internal compressions, of $\sim 1 \%$, but larger surface relaxations of $2-3 \%$.

In Figs. 4-6, we present the one-electron densities-of-states (DOS) for $\mathrm{Ru}, \mathrm{Pd}$, and $\mathrm{Ag} 135-$ atom clusters, respectively. (These were produced from the LDA eigenvalues using Gaussian broadening with an exponent of $\mathrm{E}^{2} / 4 \mathrm{a}^{2}$, with $\mathrm{a}=0.01 \mathrm{Ry}$.) The plots are quite similar to DOS from bulk systems. There are small differences in icos vs. fcc DOS. These differences are consistent with the differences in total energy, with the center of gravity being lower in the icos DOS. The "oscillations" in the DOS computed above the "d-band" for silver is a finite-size effect of the cluster.

Among the most important of the competing factors determining the optimal structure are the number and coordination of surface atoms, and the interatomic spacings of those atoms compared to ideal bulk spacings. On the fcc clusters, the surface atoms relax strongly to make up some of the energetic cost of reduced coordination. Indeed, the fcc surface atoms are more closely spaced on the finite clusters than they are on extended surfaces, demonstrating a large "surface tension" effect. For the icos clusters, however, the atoms at the surface are unable to approach each other as closely as on the fcc clusters, because of large core compressions which carry a correspondingly large energetic cost. Not requiring large core compressions to approach bulk spacings, the fcc surface atoms have more freedom to relax.

In Table 11, we show the number of atoms of each coordination and the average coordination for each cluster. While maximizing the average coordination may generate the optimal structure within the fcc class of clusters, the same need not be true in comparing fcc clusters to icos clusters because of the extra cost of compressing the core atoms in icos clusters. Nonetheless, there is a strong correlation between the average coordination of each cluster, as shown in Table 11, and the relative stability of the different structures, as in Table 2 . The anomalous behavior of $\mathrm{Ru}$ can be rationalized by comparing the above observation with surface energies of $\mathrm{Ru}$ as computed by Methfessel [29]. Open (low coordination) surfaces have much higher energies than close-packed surfaces. The bonding in $\mathrm{Ru}$ is more localized and stronger than in either $\mathrm{Pd}$ or $\mathrm{Ag}$, hence, the icos 
form for the 135-atom clusters is more strongly favored than for the 55-atom cluster because reducing the average coordination for $\mathrm{Ru}$ incurs a greater energetic penalty. With the same average coordination, as in the 140-atom clusters, the fcc form wins in two of three cases, despite having more surface atoms.

Icosahedral structures have been suggested to become unfavorable for cobalt clusters of just about this size: loss of icos signature was reported for more than 120 atoms [30]. For 140 atom clusters, the fcc geometry is already favored for $\mathrm{Ru}$ and $\mathrm{Pd}$, and this preference for fcc is likely to extend to slightly smaller clusters. The least-coordinated surface atoms of both fcc and icos structures have the same coordination and, therefore, their removal is not likely to affect the relative stabilities of icos and fcc clusters. If there is a clean transition between the icos and fcc forms for $\mathrm{Ru}$ and $\mathrm{Pd}$, it is likely to occur between 135 and 140 atoms. On the other hand, icos-based structures for Ag are likely to be more stable than fcc well beyond the next major shell closing at 147 atoms, as the icos is more stable at 140 atoms, and atoms can be added in higher coordination sites on the icos than on the fcc cluster.

It would be useful to be able to estimate energy differences between clusters as a function of the number of atoms of each coordination. With the surface energies per atom, known for many 4d transition metals including Ag [29], one could, in principle, predict the cohesive energy per atom with respect to the bulk atoms. However, using extended-surface energies to estimate cluster surface energies may not be reliable because the cluster surface nn spacings are so markedly different from those seen on bulk surfaces, especially for fcc structures. A direct computation of atom surface energies in these clusters might be more useful. For a given size of cluster, one could estimate of the relative stabilities of fcc clusters differing only in the character of surface faceting, but would not be able to compare fcc and icos clusters because of the additional need to consider the effect of core compression in the latter.

It should be noted that the geometries predicted here will not necessarily be those observed in experiments. Our calculations are for gas-phase species, while mass production techniques involve some sort of precursor in solution that either reacts or serves to nucleate growth. In the 
inverse micelle method, for example, the metal cluster grows by reducing a metal-salt cluster, surrounded by surfactant molecules [4]. The more open surfaces of bulk-like clusters, vis-a-vis icos clusters, could bind surfactants more strongly, thus generating a more stable complex despite the icos species being preferred when clean. This has also been also noted for very small metal clusters [31].

\section{QUEST Conclusions}

The transition from icos geometries, favored by small clusters, to fcc structures is predicted to occur for clusters with 140, or a few less, atoms for Ru and Pd. The icos-fcc transition for Ag is likely to occur for a much greater number of atoms. Our study shows that Harris functional calculations might be adequately accurate for $\mathrm{Ag}$ to study this transition, but that it is inadequate for systems with unclosed d-shells. Icos structures exhibit large core compressions in order to improve the interatomic spacings of the surface atoms. The fcc structures exhibit substantial surface compressions, larger than seen for extended surfaces. Hence, small nanoclusters may be expected to have surfaces with unique reactive properties - not just for morphological reasons, but also because the substantial surface relaxations suggest altered reactivity even on fcc clusters. 


\section{Studies of Small Pd and Cu Nanoclusters Using the DMOL Code}

\section{Self-Consistent Convergence Using DFT}

It was found early on that it was possible to converge our density functional calculations for both $\mathrm{Cu}$ and $\mathrm{Pd}$ clusters. Self-consistent convergence was attained by using a combination of techniques. In general, it was necessary to "smear" out the occupation of electronic states near the Fermi energy - in effect by performing the calculations at a nonzero Fermi temperature. In addition it was necessary to provide a mixing factor, which blended solutions from iteration to iteration. For certain calculations a very low mixing factor was required, leading to very slow convergence. This was done using the capabilities built into the DMOL commercial density functional code of Biosym Technologies, Inc. The built-in geometry optimization tools in DMOL also proved valuable for sorting out optimal geometries for clusters of various sizes.

\section{13-Atom Bare Clusters}

For both $\mathrm{Pd}$ and $\mathrm{Cu}$, calculations on 13-atom icosohedral clusters were performed, see Figure 1. The icosohedral symmetry was surmised to be the likely optimal geometry for these clusters based on various experimental results by other research groups (later on we were able to check this computationally - see section 3). The converged geometries for both $\mathrm{Pd}$ and $\mathrm{Cu}$ showed some interesting features. The interatomic distances were reduced compared with bulk values. In particular, there was considerable "squeezing" of the central atom in the icosohedran - that is, the interatomic distance between the central atom and outer atoms was reduced with respect to bulk interatomic values. For $\mathrm{Cu}$ there was a reduction of about $8 \%$ and for $\mathrm{Pd}$ the reduction was about $4 \%$. The

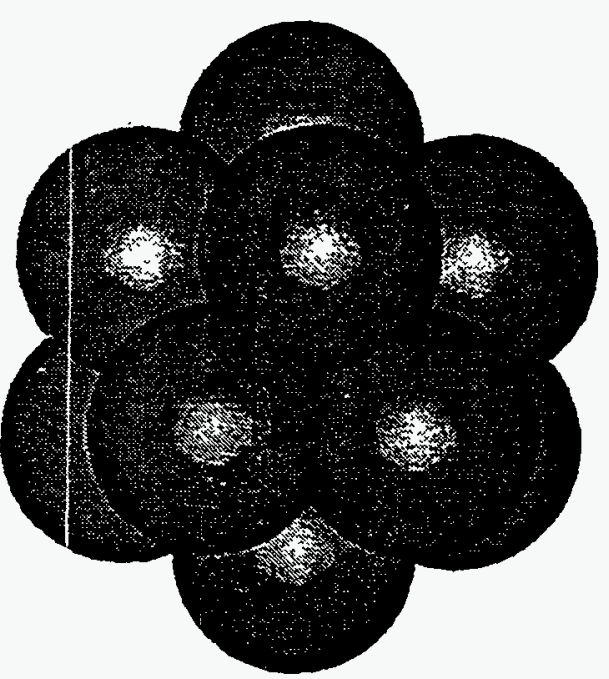

Figure 1: 13-atom Icosohedral Cu Cluster. 
interatomic distance between outer shell atoms was also reduced, but by lesser amounts. For $\mathrm{Cu}$ this reduction was about $3 \%$ and for $\mathrm{Pd}$ it was about $1.5 \%$. Electronically, the calculations showed multiple degeneracies at the Fermi Level, indicating that breaking the geometric symmetry of the clusters should lower the total energy (Jan Teller Distortions). However, despite repeated attempts using various approaches, we were unable to find distortions of the icosohedral structure which lead to more stable structures than the fully symmetric geometry.

\section{Other Non-Icosohedral Geometries}

To check the assertion that the icosohedral geometry is lower in energy than other geometries, we performed calculations on 13-atom $\mathrm{Cu}$ clusters with hexagonal close packed (HCP) - Figure 2, Face Centered Cubic (FCC) - Figure 3, and the so-called Optet

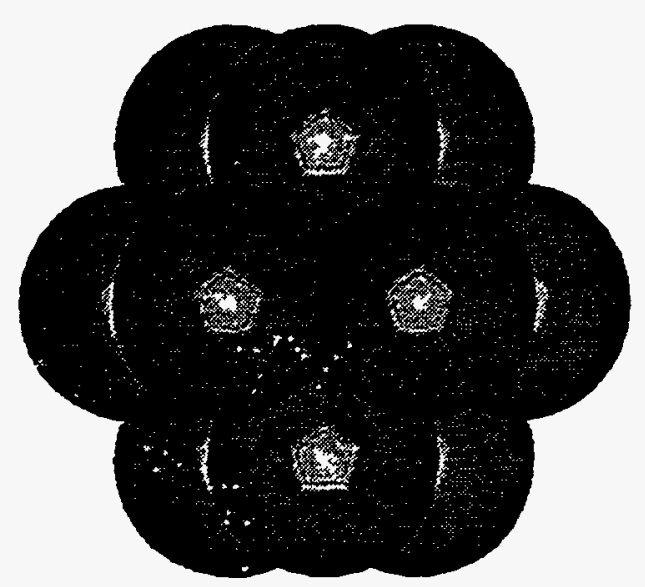

Figure 2: 13-atom $\mathrm{HCP} \mathrm{Cu}$ Cluster.

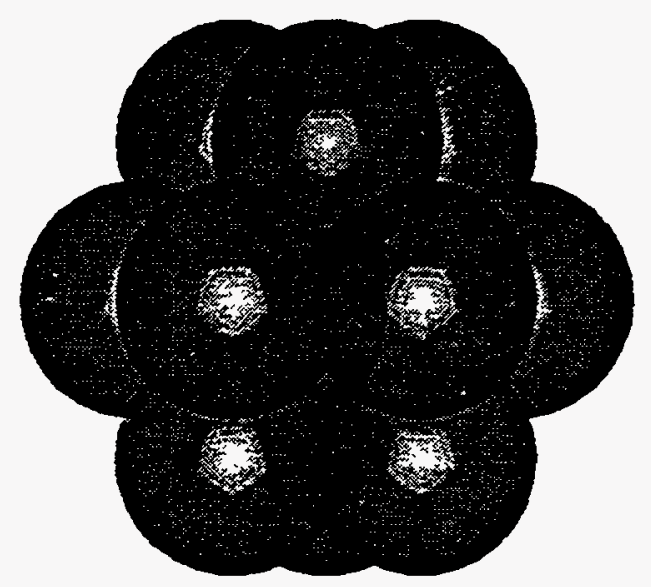

Figure 3: 13-atom FCC Cu Cluster.

structure proposed by Goddard for Alkali metals - Figure 4. For the bulk-like geometries, $\mathrm{HCP}$ and FCC, it was found that the icosohedral geometry had a higher total binding energy no matter how the calculations were performed (with or without polarization functions, with or without spin restrictions). However, for the optet structure, the results depended strongly on the treatment of electronic properties, particularly the proper treatment of the spin. For instance, in a spin-restricted calculation with no additional polarization functions over the standard DMOL basis set, the total binding energy for $\mathrm{HCP}$ was $33.4 \mathrm{eV}$, for FCC was $34.0 \mathrm{eV}$, for icosohedral was $34.1 \mathrm{eV}$, and for the Optet was $34.6 \mathrm{eV}$. Indicating that while the icosohedral geometry was more stable than the bulk-like geometries, it was less favorable than the Optet geometry. Adding polarization functions did not change this ordering although the binding energies increased by about $0.1 \mathrm{eV}$ across the board. Performing spin-unrestricted calculations, however, changed this situation. In this case, the Optet binding energy increased to $34.75 \mathrm{eV}$ while the icosohedral geometry had its calculated total binding energy increase dramatically to $34.80 \mathrm{eV}$, indicating once again that the

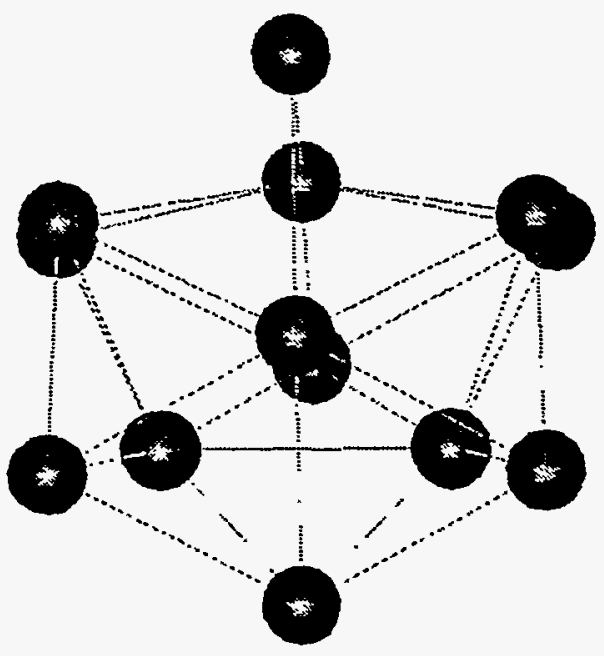

Figure 4: 13-atom Optet cluster. 
icosohedral geometry is most favorable (albeit by a small amount). Adding adsorbates to these structures further increases the favorability of the icosohedral geometry (see section 4).

\section{Hydrogen Adsorption}

a) Single Hydrogen Atom Adsorption Studying the binding of simple molecules on these clusters is the first step towards developing an understanding of the chemical properties of these nanocluster structures. For this purpose we studied the binding of hydrogen atoms on the $\mathrm{Cu}$ and $\mathrm{Pd}$ 13-atom icosohedral clusters. For both $\mathrm{Cu}$ and $\mathrm{Pd}$ it was found that the atop site and the two-fold bridge sites were unstable with respect to the 3-fold hollow site - Figure 5. In addition, the binding energies for hydrogen were quite similar - about $3.4 \mathrm{eV}$ for both $\mathrm{Cu}$ and $\mathrm{Pd}$. The addition of hydrogen also caused a relaxation in the clusters, with the interatomic distances between the central atom and the 3 atoms proximate to the hydrogen atom relaxing to their bulk values. For the $\mathrm{Cu}$ Optet structure, hydrogen has a binding energy of only $2.9 \mathrm{eV}$, further reducing its favorability as a possible physical structure.

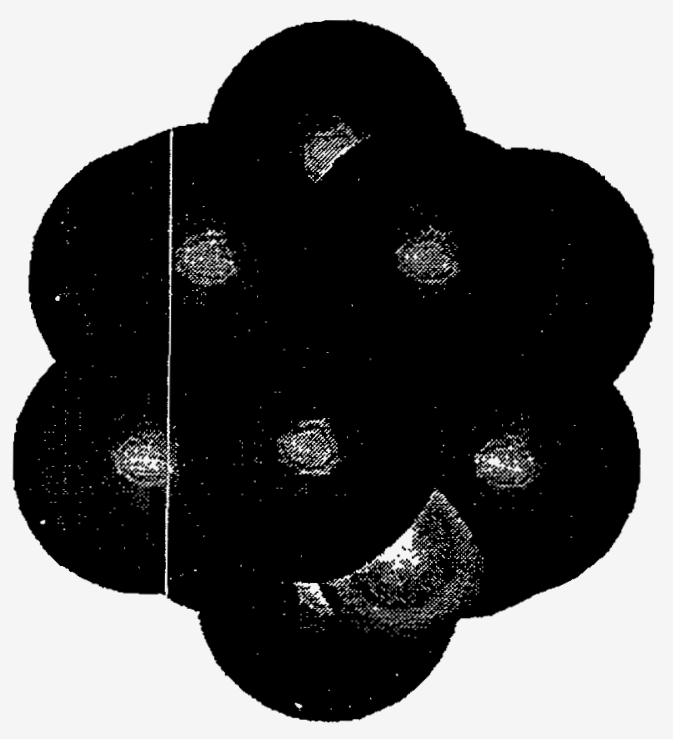

Figure 5: 13-atom icosohedral PdCluster with Hydrogen adsorbed at the 3-fold hollow site.

\section{b) Multiple Hydrogen Atom Adsorption}

The situation becomes more interesting as multiple hydrogen atoms are placed on the surface with various separations. Contrary to what one might expect, it appears there is very little interaction between hydrogen atoms in 3-fold sites. No matter what the separation between two adsorbed H-atoms - Figures $6-8$, it was found that the binding energy per hydrogen atom was nearly identical to that of a single hydrogen atom bound to the cluster, $3.35 \mathrm{eV}$. The one exception to this was when the two hydrogen atoms were placed diametrically opposite to each other - Figure 9 . In this case the binding energy per hydrogen atom dropped to $2.95 \mathrm{eV}$. This is undoubtedly due to the degeneracies at the Fermi level that this symmetric situation produces and probably does not have physical significance.

As more hydrogen is added to the surface the binding energy per hydrogen atom decreases very slowly, further emphasizing the lack of interactions between individual bound hydrogen atoms. For instance, a loading of 10 hydrogen atoms has a binding energy of $3.06 \mathrm{eV}$ per hydrogen atom - Figure 10. Eventually, however the binding energy of the hydrogen atoms to the $\mathrm{Cu}$ cluster drops below that of hydrogen to itself (LDA gives a total binding energy of about $4.5 \mathrm{eV}$ for the hydrogen molecule, or $2.3 \mathrm{eV}$ per hydrogen atom). At this point, the cluster becomes unstable with respect to the formation of hydrogen molecules. A fully loaded cluster (20 hydrogen atoms in all available 3-fold sites - Figure 11) has a binding energy somewhat less that $2.0 \mathrm{eV}$ per hydrogen. Calculations show the entire cluster distorting dramatically with hydrogen molecules forming and presumably desorbing from the cluster - Figure 12. 


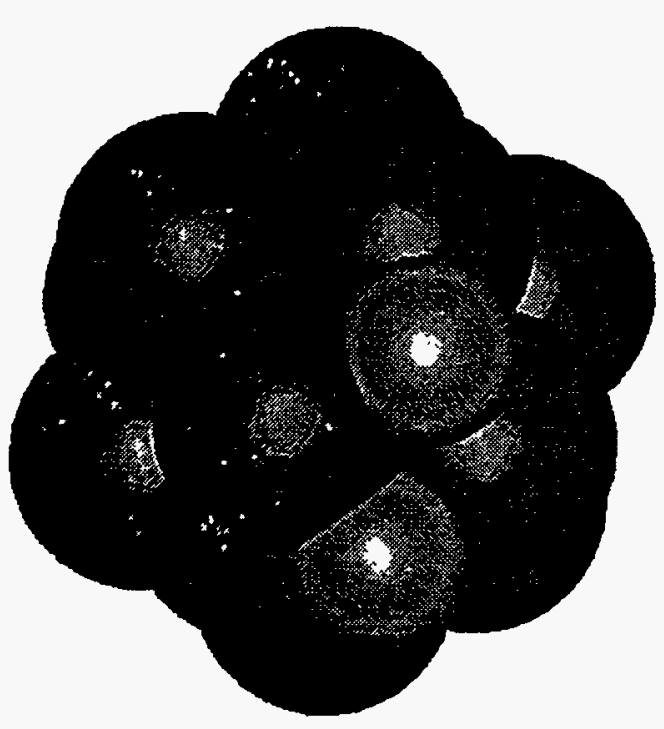

Figure 6: 13-atom icosohedral $\mathrm{Cu}$ Cluster with hydrogen atoms at neighboring 3-fold sites.

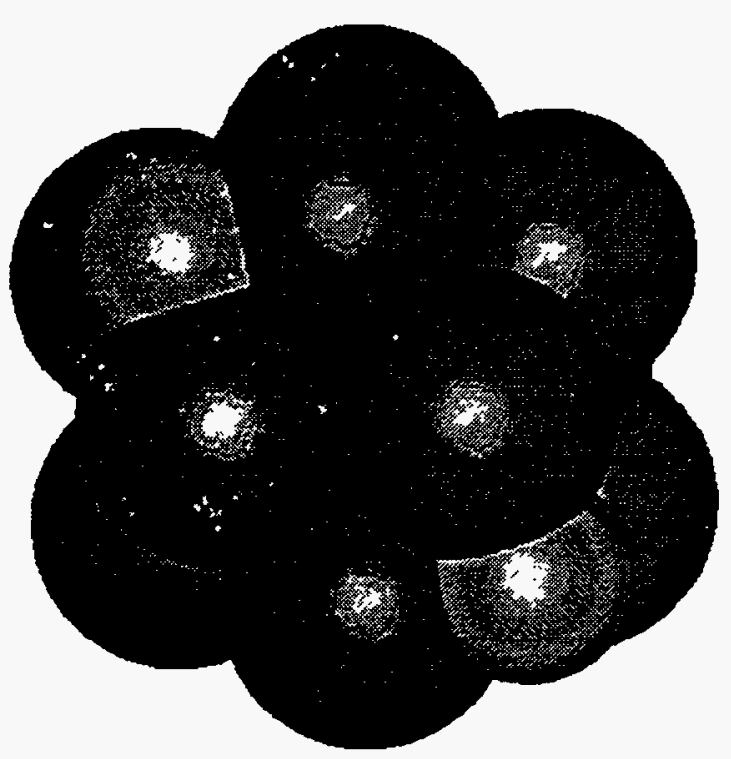

Figure 8: 13-atom icosohedral $\mathrm{Cu}$ cluster with hydrogen atoms spaced 3 sites apart.

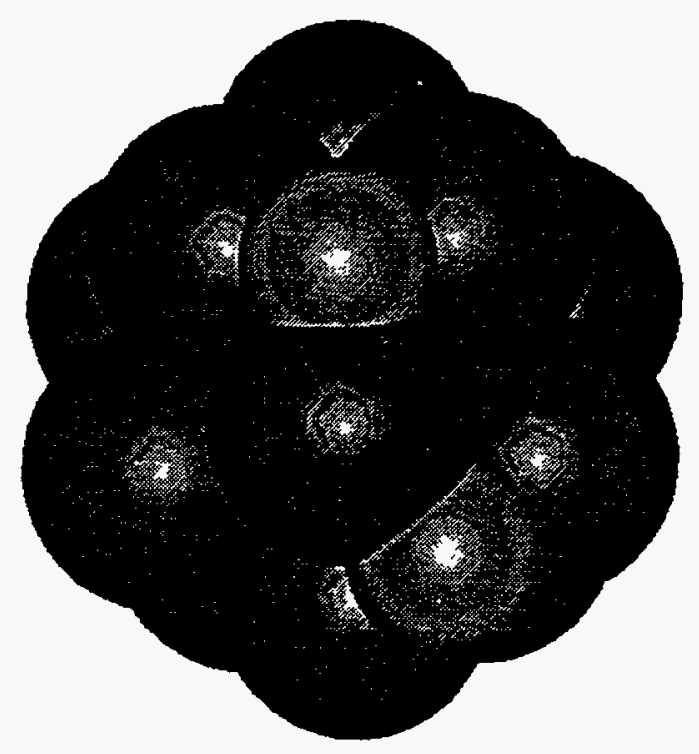

Figure 7: 13-atom icosohedral $\mathrm{Cu}$ cluster with hydrogen atoms spaced 2 sites apart.

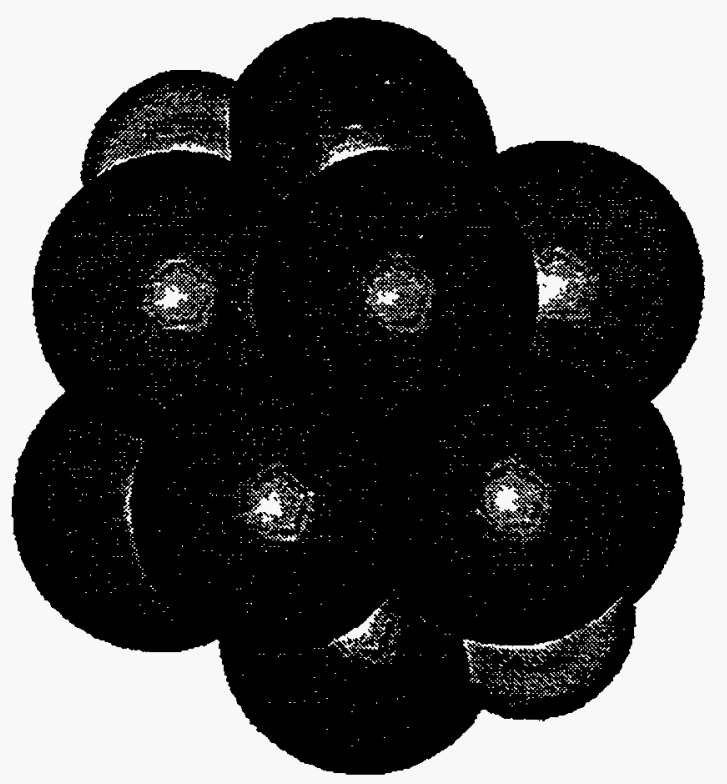

Figure 9: 13-atom icosohedral $\mathrm{Cu}$ cluster with hydrogen atoms diametrically opposite each other.

We did find one curious structure that appeared stable. 20 hydrogen atoms were placed at 3 -fold hollow sites and allowed to diffuse to the center of the icosohedral cluster. This pushed the 12 outer atoms away from the central atom and appeared to form a stable (though perhaps unphysical) structure - Figure 13. 


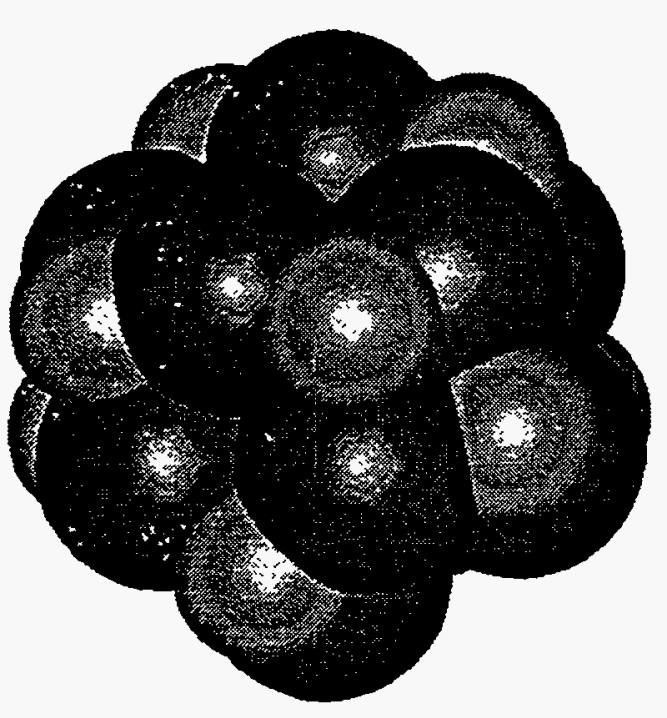

Figure 10: 13-atom icosohedral $\mathrm{Cu}$ cluster with 10 hydrogen atoms.

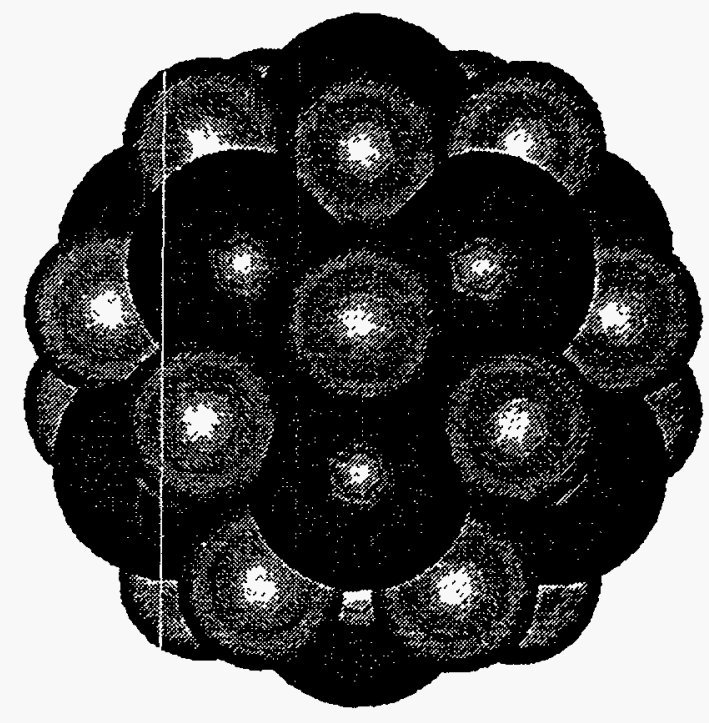

Figure 11: 13-atom icosohedral $\mathrm{Cu}$ cluster with 20 hydrogen atoms (initial non-optimized geometry).

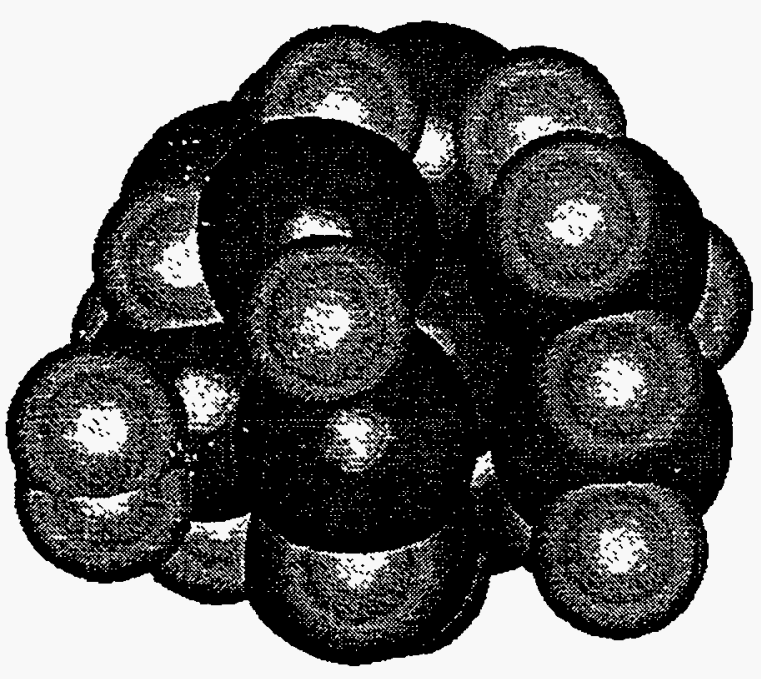

Figure 12: 13-atom icosohedral $\mathrm{Cu}$ cluster with 20 hydrogen atoms (after some optimization).

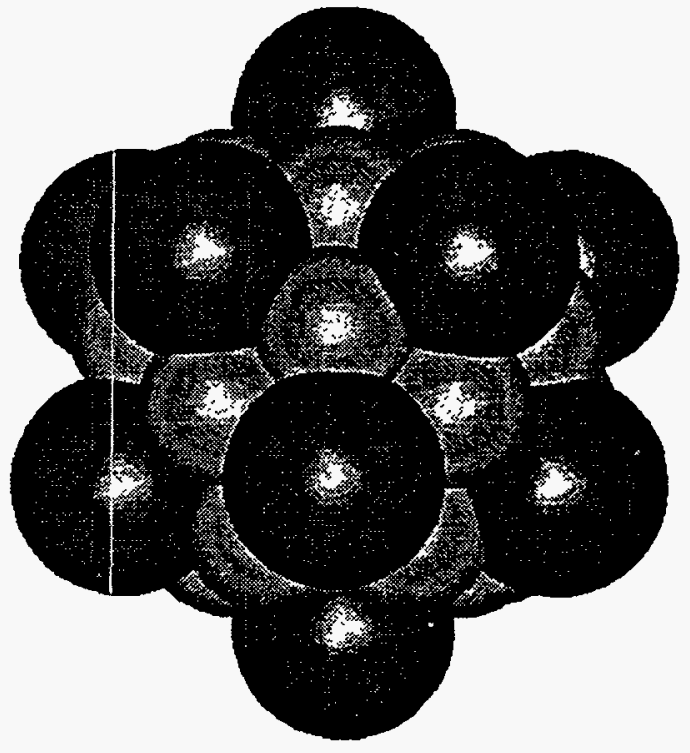

Figure 13: 13-atom icosohedral $\mathrm{Cu}$ cluster with 20 hydrogen atoms (after allowing the hydrogen to diffuse beneath the outer shell).

\section{Summary}

We have shown that it is possible to study coinage and transition metal clusters using first principles DFT. It was found that the proper treatment of spin was important in determining the optimal geometry for these relatively small clusters. Adsorption of 
hydrogen yielded insight into the stable binding sites on these clusters, the relative interaction between these sites, and possible changes in geometry due to adsorbates. While the lack of interaction between hydrogen binding sites allows relatively large quantities of hydrogen to adsorb onto a small cluster (suggesting a possible use for nanocluster materials for hydrogen storage applications), at the highest loadings these clusters become unstable with respect to molecular hydrogen formation. These studies suggest that it is indeed possible to use DFT as a tool for understanding the novel physical and chemical properties these metal nanoclusters possess. 


\section{Conclusion}

Goals which we have reached include solving convergence problems associated with large transition metal clusters, which have a large number of eigenstates close to the Fermi level; performing transition metal cluster calculations of record size; and obtaining the relaxed geometries and relative energies of clusters ranging from 13 to 140 transition metal atoms.

A goal not reached is the study of transition states for simple chemical reactions on cluster surfaces. During the course of this study, it became widely known that standard LDA theory is insufficient to compute transition states for many systems. The gradient correction to standard LDA (so-called GGA) has been shown for some systems to solve this failing. However, other recent work has raised the suspicion that even GGA cannot obtain qualitatively accurate transition states for some systems. A solution is to use LDA to obtain geometries, but, before transition state calculations are carried out, compare LDA and GGA to more accurate first principles methods such as quantum Monte Carlo for a system of interest, modeled by a small cluster; this way, the intrinsic inaccuracies in the density functional methods may be learned and reliability of results established. 


\section{References}

1. For a review, see B. H. S. Thimmappa, Coord. Chem. Revs. 143, 1 (1995), and references therein.

2. M. T. Reetz and W. Helbig, J. Am. Chem. Soc. 116, 7401 (1994).

3. For example, M. Brust, M. Walker, D. Bethell, D. J. Schiffrin, and R. Whyman, J. Chem. Soc. Chem. Comm. 801 (1994).

4. A. Martino, J. P. Wilcoxon, A. P. Sylwester, and J. S. Kawola, Am. Chem. Soc., Div. Fuel Chem. 38, 20 (1993); J. P. Wilcoxon, T. Martino, E. Klavetter, and A. P. Sylwester, NATO ASI Ser. E 260 (Nanophase Materials), 771 (1994).

5. See the special issue of Science magazine, 16 February 1996.

6. A. Martino, J. P. Wilcoxon, J. S. Kawola, Energy Fuels 8, 1289 (1994).

7. E. K. Parks, et al., J. Chem. Phys. 88, 1662 (1988); P. S. Bechthold, et al., Z. Phys. Chem. N. F. 169, 101 (1990).

8. H. Handschuh, G. Gantefor, and W. Eberhardt, Rev. Sci. Inst. 66, 3838 (1995); G. Gantefor and W. Eberhardt, Chem. Phys. Lett. 217, 600 (1994); C. Y. Cha, G. Gantefor, and W. Eberhardt, J. Chem. Phys. 99, 6308 (1993).

9. H. N. Aiyer, V. Vijayakrishnan, G. N. Subbanna, and C. N. R. Rao, Surf. Sci. 313, 392 (1994).

10. J. C. Grossman and L. Mitas, Phys. Rev. Lett. 74, 1323 (1995); Phys. Rev. B 52, 16735 (1995).

11. E. K. Parks, L. Zhu, J. Ho, and S. J. Riley, Zeit. Phys. D 26, 41 (1993).

12. E. K. Parks, B. J. Winter, T. D. Klots, and S. J. Riley, J. Chem. Phys. 96, 8267 (1992).

13. EAM predicts an inward relaxation of 0.2 a.u. for the top layer of $\mathrm{Pd}$ and $\mathrm{Pt}$ (111); LDA predicts a small outward relaxation of 0.04 a.u.; D. R. Jennison and J. C. Hamilton, unpublished.

14. Written and developed by M. P. Sears and P. A. Schultz at Sandia National Laboratories, Albuquerque, NM 87185-1111.

15. P. Hohenberg and W. Kohn, Phys. Rev. 136, B864 (1964).

16. W. Kohn and L. J. Sham, Phys. Rev. 140, A1133 (1965); see also Theory of the Inhomoge- 
neous Electron Gas, edited by S. Lundqvist and N. M. March (Plenum, New York, 1983).

17. P. J. Feibelman, Annu. Rev. Phys. Chem. 40, 261 (1989).

18. J. Perdew, et al., Phys. Rev. B 46, 6671 (1992) and ibid., submitted.

19. J. Perdew and A. Zunger, Phys. Rev. B 23, 5048 (1981).

20. D. M. Ceperly and B. J. Alder, Phys. Rev. Lett. 45, 566 (1980).

21. D. R. Hamann, Phys. Rev. B 40, 2980 (1989).

22. A. J. Cox, J. G. Louderback, S. E. Apsel, and L. A. Bloomfield, Phys. Rev. B 49, 12295 (1994).

23. For more information contact P. A. Schultz, Sandia National Laboratories, Albuquerque, NM 87185-1111, paschul@cs.sandia.gov; see also P. J. Feibelman, Phys. Rev. B 38, 1849 (1988).

24. P. J. Feibelman, Phys. Rev. B 33, 719 (1986); ibid., 35, 2626 (1987).

25. P. Pulay, Mol. Phys. 17, 197 (1969).

26. D. D. Johnson, Phys. Rev. B 38, 12807 (1988).

27. J. Harris, Phys. Rev. B 31, 1770 (1985).

28. S. H. Yang, D. A. Drabold, J. B. Adams and A. Sachdev, Phys. Rev. B 47, 1567 (1993).

29. M. Methfessel, D. Henning, and M. Schleffer, Phys. Rev. B 46, 4816 (1992).

30. E. K. Parks, T. D. Klots, B. J. Winter, and S. J. Riley, J. Chem. Phys. 99, 5831 (1993); see also E. K. Parks, L. Zhu, J. Ho, and S. J. Riley, Z. Phys. D 26, 41 (1993).

31. V. G. Albano, et al., J. Chem. Soc., Chem. Commun., 859 (1975); also see the discussion in Ref. 1. 


\section{Appendix}

\section{Publications:}

$A b$ initio calculations of $\mathrm{Ru}, \mathrm{Pd}$, and $\mathrm{Ag}$ cluster structure with 55,135 and 140 atoms, D. R. Jennison, P. A. Schultz, and M. P. Sears, J. Chem. Phys., submitted.

Chemisorbed-molecule potential energy surfaces and DIET processes, Proceedings of the 6th International Workshop on Desorption Induced by Electronic Transitions, Nucl. Inst. Meth. Phys. Res. B 101, 22 (1995).

\section{Presentations:}

A Giant Leap in First-Principles Computational Surface Science, D. R. Jennison, P. A. Schultz, and M. P. Sears, invited seminar, Univ. of Liverpool, UK, 6/28/96.

First Principles Studies of 55- and 135-Atom Clusters of Ru, Pd, and Ag, D. R. Jennison, P. A. Schultz, and M. P. Sears, Americal Physical Society March Meeting, 3/18/96.

Nanocluster Surface Science by LDA Theory, D. R. Jennison, Y. S. Li, P. A. Schultz, M. P. Sears, T. Klitsner, and P. Feibelman, American Vacuum Society 41st National Symposium, 10/25/ 94. 
Fig. 1. The 55-atom fcc and icosahedral clusters.
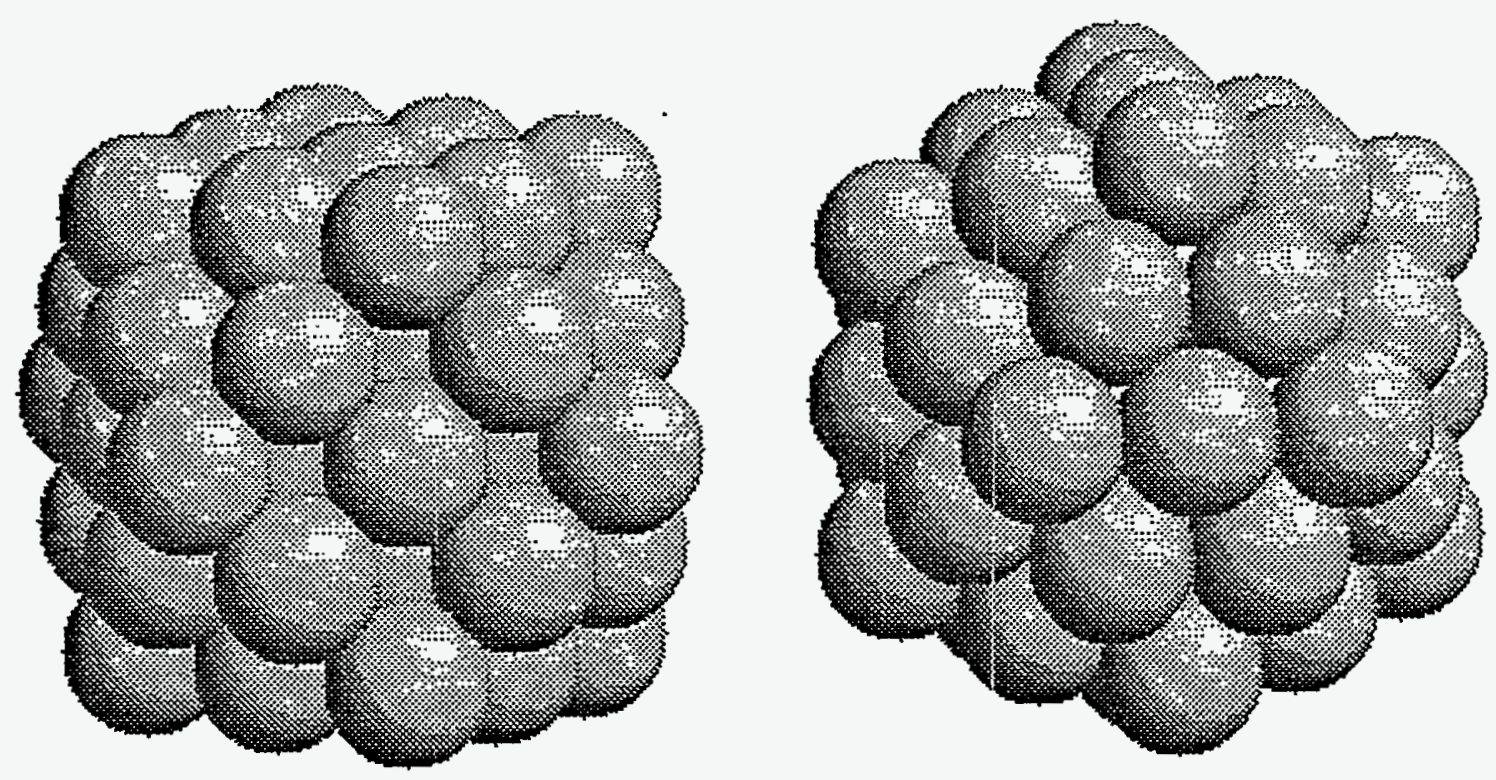
Fig. 2. The 135-atom fcc and icosahedral clusters.
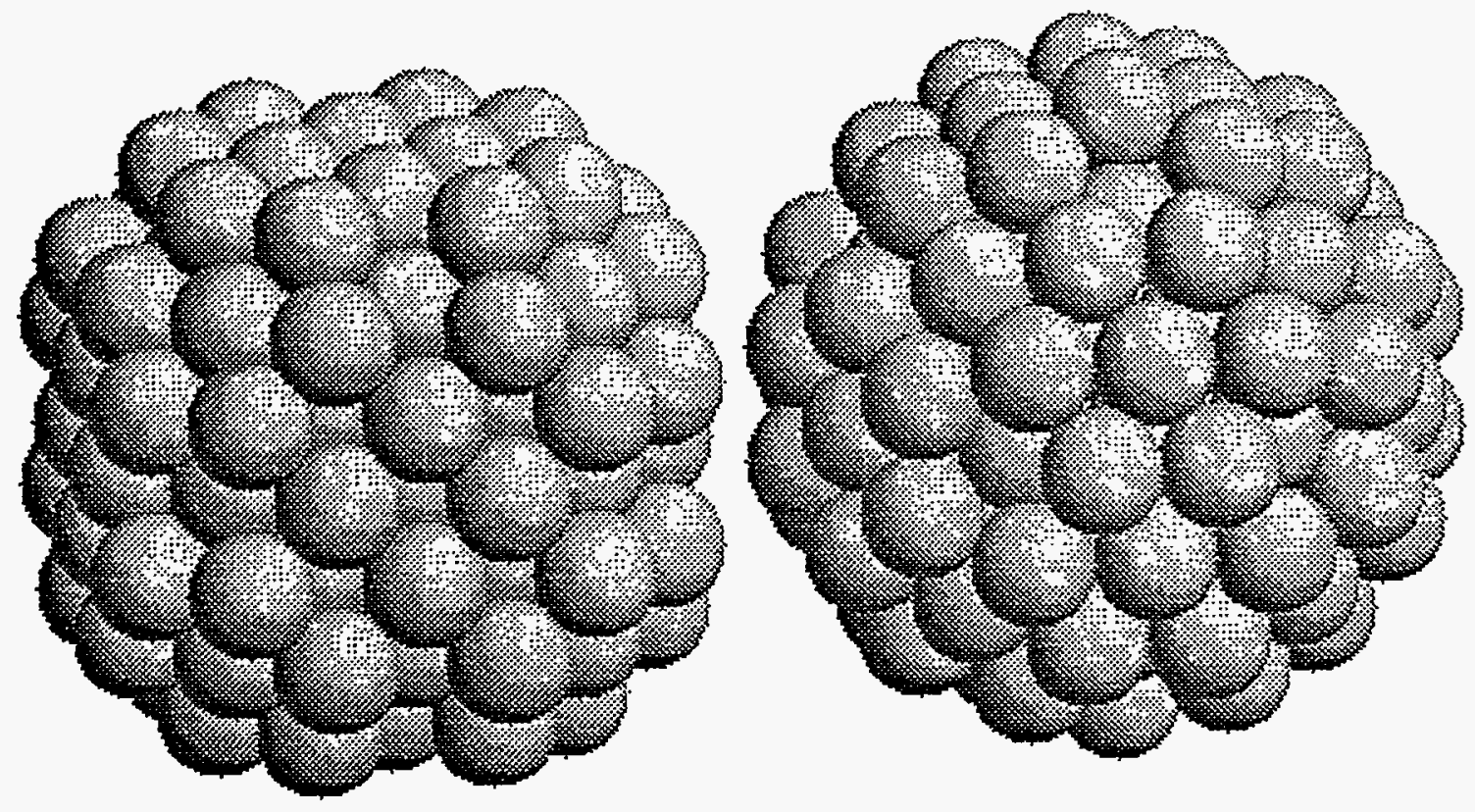
Fig. 3. The 140-atom fcc octahedron.

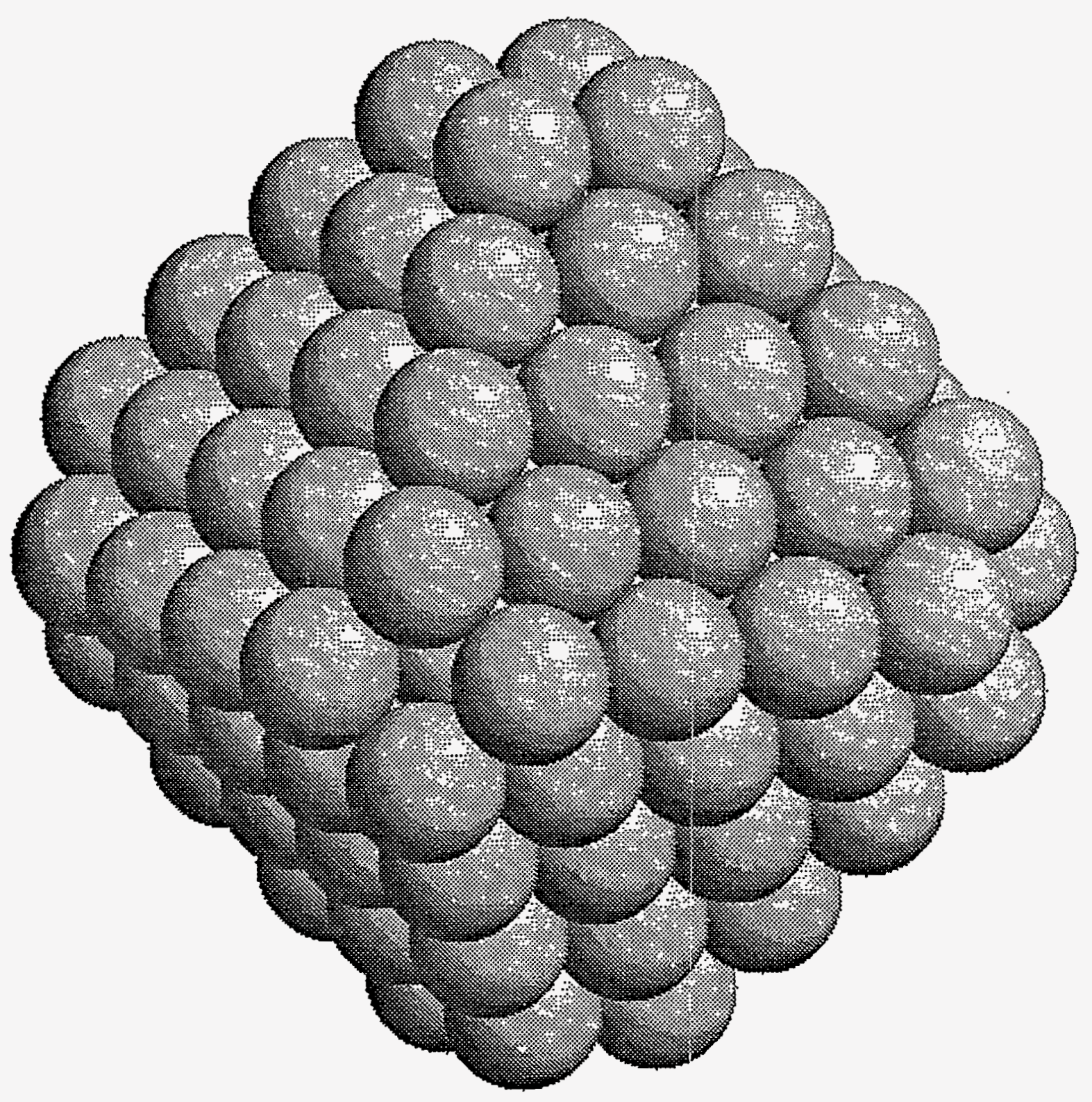


Fig. 4. The DOS of the 135 atom icosahedral (solid) and fcc (dashed) Ru clusters.

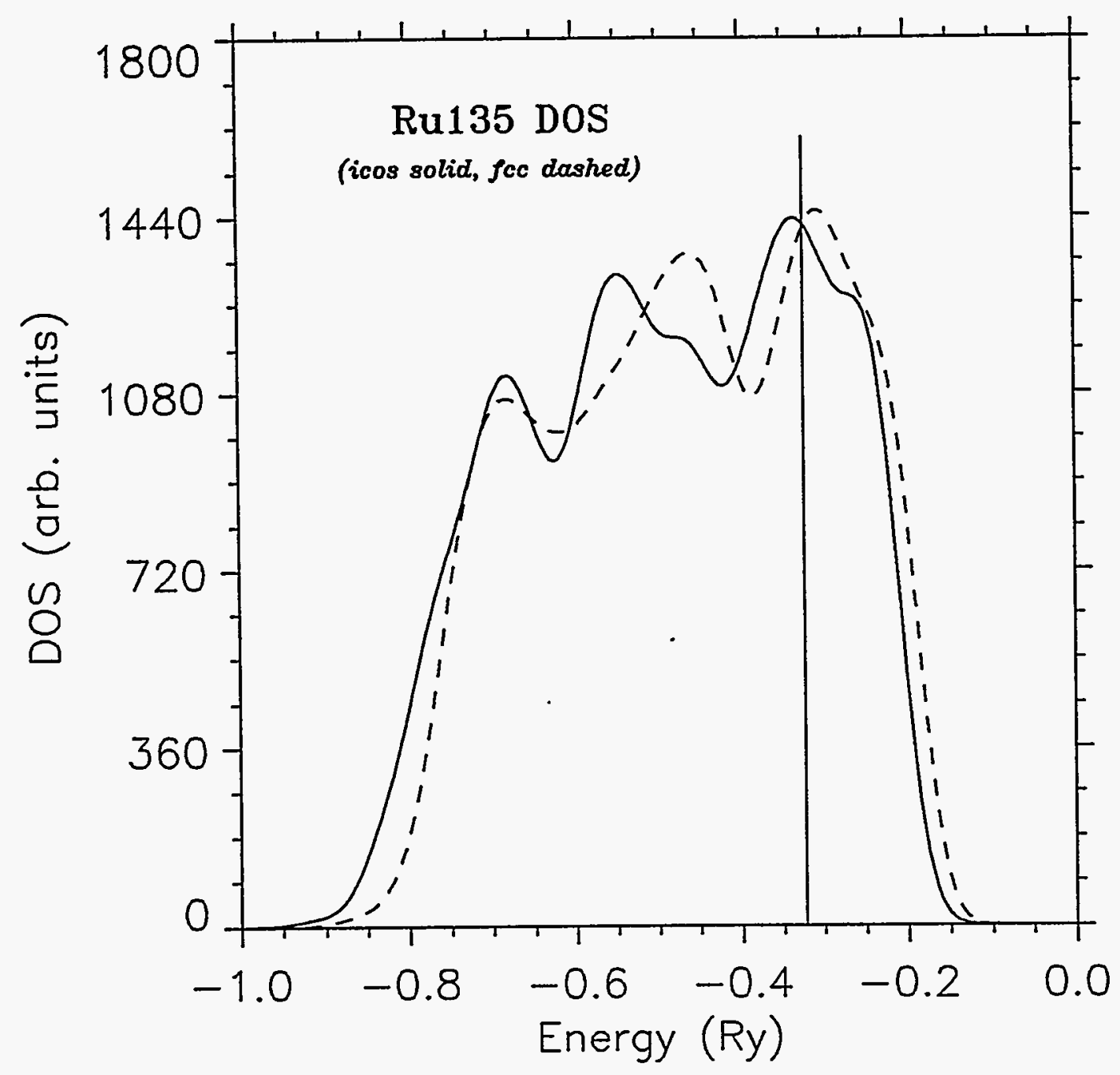


Fig. 5. The DOS of the 135 atom icosahedral (solid) and fcc (dashed) Pd clusters.

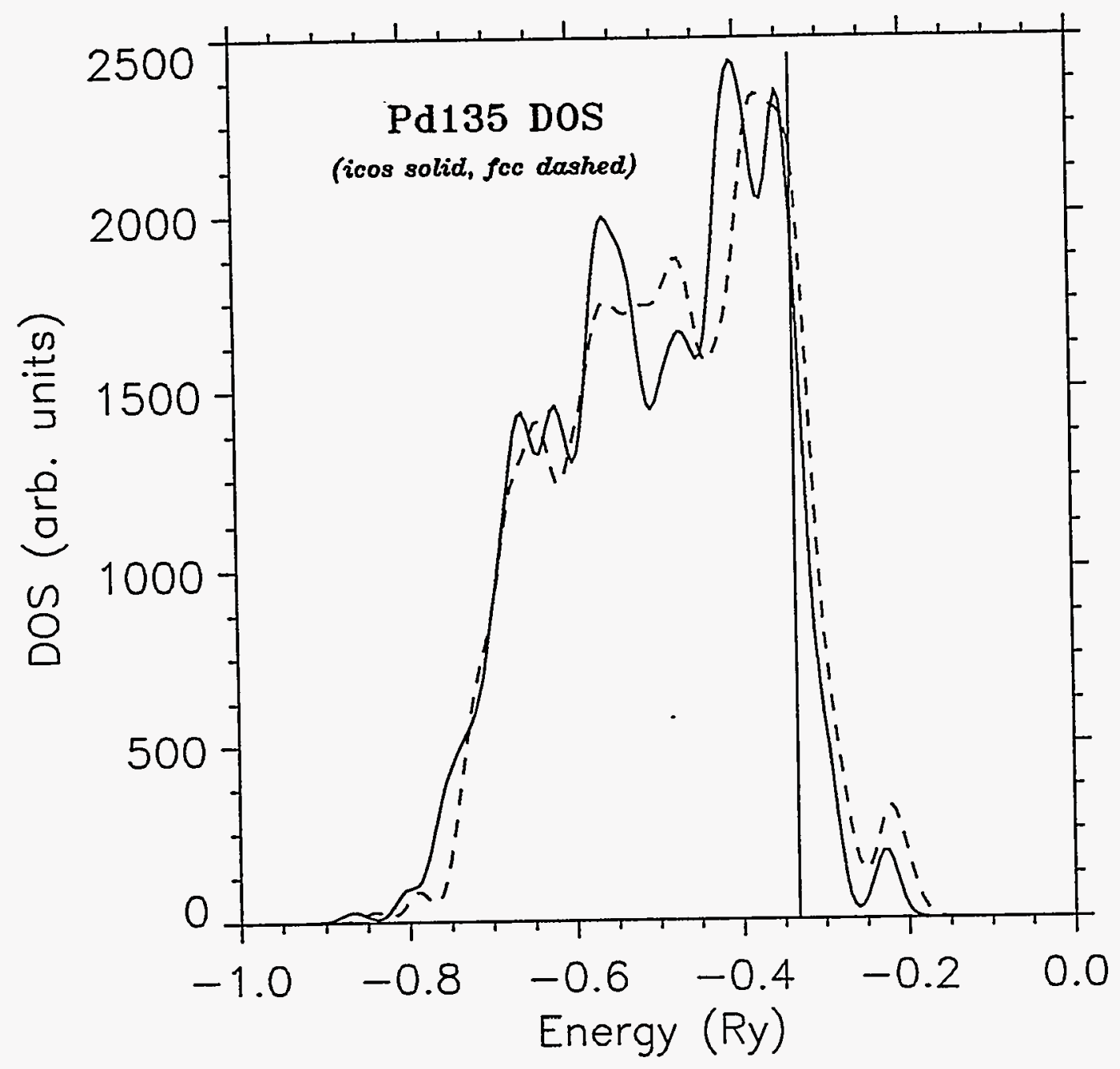


Fig. 6. The DOS of the 135 atom icosahedral (solid) and fcc (dashed) Ag clusters.

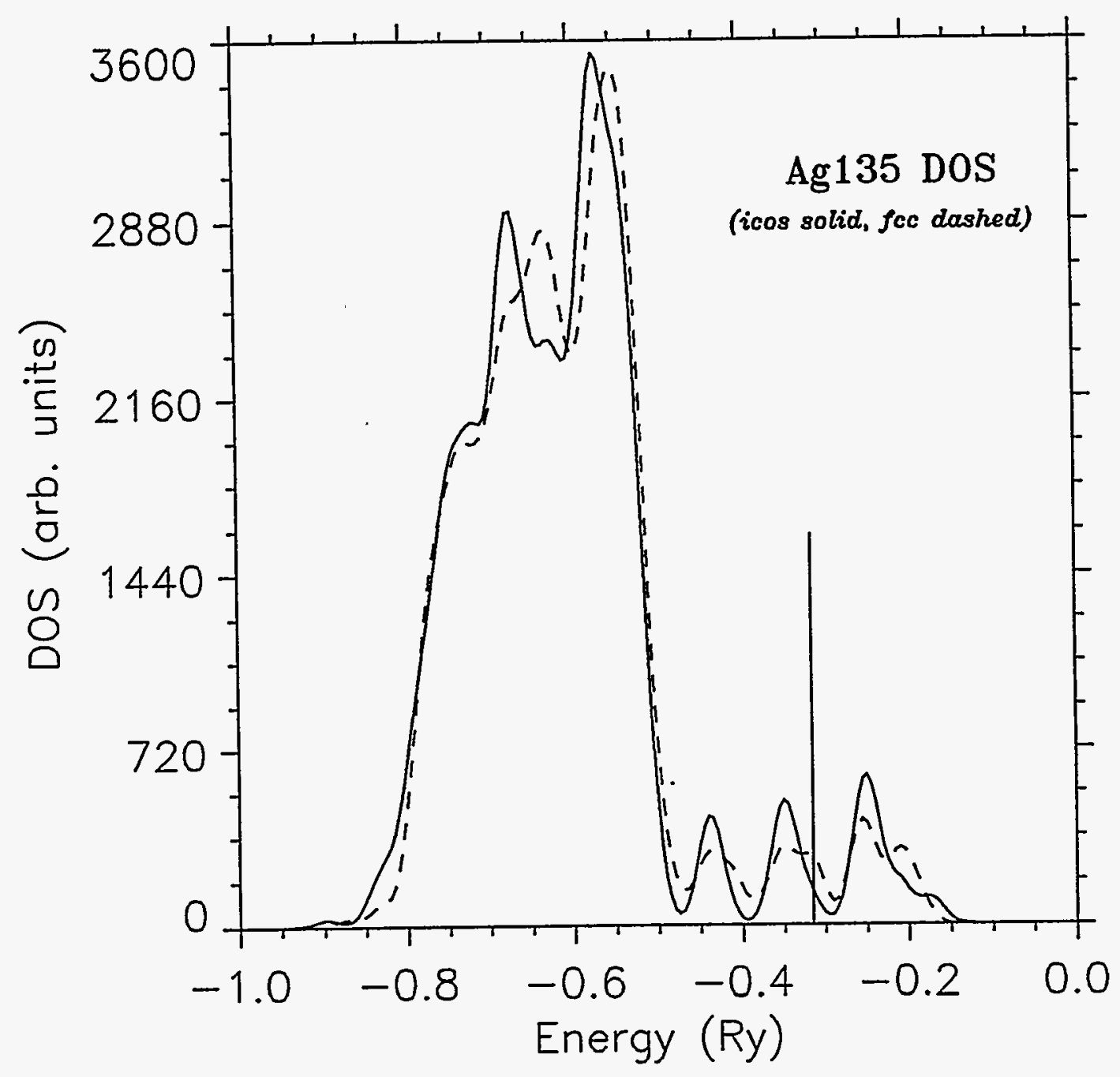




\section{Tables}

Table 1: Basis set tests: "single/double zeta" (1d/2d) lattice constant $a_{0}$, bulk modulus $B$, energy per atom E/At

\begin{tabular}{|c||c|c|c|}
\hline Basis: & $\mathrm{Ru}(\mathrm{fcc})$ & $\mathrm{Pd}$ & $\mathrm{Ag}$ \\
\hline \hline $\mathrm{a}_{\mathrm{o}}$ (au): & $7.074 / 7.042$ & $7.279 / 7.274$ & $7.570 / 7.581$ \\
\hline $\mathrm{B}$ (Mbar): & $3.76 / 3.45$ & $2.34 / 2.25$ & $1.57 / 1.47$ \\
\hline $\mathrm{E}$ (Ry)/At: & $-33.1596 /$ & $-58.3176 /$ & $-74.4705 /$ \\
& .1622 & .3181 & .4715 \\
\hline
\end{tabular}

Table 2: Energy differences per atom (i.e., total energy difference divided by the number of atoms, $\mathbf{N}$ ) of the fcc with respect to icosahedral structures (in $\mathrm{eV}$ ).

\begin{tabular}{|c||c|c|c|}
\hline Material: & $\mathrm{Ru}$ & $\mathrm{Pd}$ & $\mathrm{Ag}$ \\
\hline \hline $\mathrm{N}=55, \Delta \mathrm{E}:$ & +0.047 & +0.019 & +0.059 \\
\hline $\mathrm{N}=135, \Delta \mathrm{E}:$ & +0.057 & +0.013 & +0.029 \\
\hline $\mathrm{N}=140, \Delta \mathrm{E}:$ & -0.035 & -0.022 & +0.029 \\
\hline
\end{tabular}

Table 3: Harris-functional total energy differences, $H$, (icosahedral minus fcc) for $N=140$ vs. full LDA SCF energy differences (SCF). "It." means geometry relaxation iteration.

\begin{tabular}{|c||c|c|c|c|c|c|}
\hline $\mathbf{N}$ & $\begin{array}{c}\text { It. 0 } \\
\text { Ru H/SCF }\end{array}$ & $\begin{array}{c}\text { It. final } \\
\text { Ru H/SCF }\end{array}$ & $\begin{array}{c}\text { It. 0 } \\
\text { Pd H/SCF }\end{array}$ & $\begin{array}{c}\text { It. final } \\
\text { Pd H/SCF }\end{array}$ & $\begin{array}{c}\text { It. 0 } \\
\text { Ag H/SCF }\end{array}$ & $\begin{array}{c}\text { It. final } \\
\text { Ag H/SCF }\end{array}$ \\
\hline \hline 140 & $0.39 / 0.23$ & $0.66 / 0.37$ & $0.06 / 0.13$ & $0.07 / 0.21$ & $-0.32 /-0.27$ & $-0.34 /-0.30$ \\
\hline
\end{tabular}


Table 4: Shell, number of shell atoms ( $n$ ), positions (ijk), and radii at bulk distances vs. relaxed fcc Ru clusters ( $\AA$ ). (LDA bulk radius 1.318 vs. 1.355 , experiment.) " $\Delta<n n\rangle "=\left\langle\mathrm{d}_{n n}>/ d^{\text {bulk }}{ }_{n n}\right.$.

\begin{tabular}{|c|c|c|c|c|c|}
\hline Shell & $\mathrm{n}(\mathrm{i}, \mathrm{j}, \mathrm{k})$ & $\mathrm{Ru}$ bulk & $\mathrm{Ru} 55$ & $\mathrm{Ru} 135$ & $\Delta<\mathrm{nn}>(\%)$ \\
\hline \hline 0. & $1(000)$ & 0.000 & 0.000 & 0.000 & -1.4 \\
\hline 1 & $12(110)$ & 2.636 & 2.608 & 2.598 & -0.25 \\
\hline 2 & $6(200)$ & 3.728 & 3.624 & 3.739 & -0.80 \\
\hline 3 & $24(211)$ & 4.566 & 4.446 & 4.559 & -1.2 \\
\hline 4 & $12(220)$ & 5.272 & 5.089 & 5.251 & -1.5 \\
\hline 5 & $24(310)$ & 5.894 & - & 5.843 & -1.4 \\
\hline 6 & $8(222)$ & 6.457 & - & 6.364 & -3.0 \\
\hline 7 & $48(321)$ & 6.974 & - & 6.821 & -3.4 \\
\hline
\end{tabular}

Table 5: Geometry of relaxed icosahedral Ru (bulk nn LDF distance is $2.636 \AA$ ). (Symbols are as in Table 2.)

\begin{tabular}{|c|c|c|c|c|}
\hline Shell & $\mathrm{n}$ & $\mathrm{Ru} 55$ & $\mathrm{Ru} 135$ & $\Delta<\mathrm{nn}>(\%)$ \\
\hline \hline 0 & 1 & 0.000 & 0.000 & -5.9 \\
\hline 1 & 12 & 2.528 & 2.480 & -2.0 \\
\hline 2 & 30 & 4.296 & 4.324 & -1.0 \\
\hline 3 & 12 & 4.947 & 5.117 & -1.2 \\
\hline 4 & 20 & - & 6.101 & -0.76 \\
\hline 5 & 60 & - & 6.566 & -1.3 \\
\hline
\end{tabular}


Table 6: Geometry of relaxed fcc Pd clusters ( $\AA$ ). (LDA bulk radius is 1.361 vs. 1.37 , experiment.)

\begin{tabular}{|c|c|c|c|c|c|}
\hline Shell & $\mathrm{n}(\mathrm{i}, \mathrm{j}, \mathrm{k})$ & Pd bulk & Pd 55 & Pd 135 & $\Delta<\mathrm{nn}>(\%)$ \\
\hline \hline 0 & $1(000)$ & 0.000 & 0.000 & 0.000 & -1.0 \\
\hline 1 & $12(110)$ & 2.722 & 2.642 & 2.698 & -1.2 \\
\hline 2 & $6(200)$ & 3.850 & 3.885 & 3.820 & -1.2 \\
\hline 3 & $24(211)$ & 4.715 & 4.613 & 4.629 & -1.1 \\
\hline 4 & $12(220)$ & 5.444 & 5.250 & 5.427 & -1.2 \\
\hline 5 & $24(310)$ & 6.087 & - & 6.080 & -1.2 \\
\hline 6 & $8(222)$ & 6.668 & - & 6.601 & -0.78 \\
\hline 7 & $48(321)$ & 7.202 & - & 7.035 & -2.4 \\
\hline
\end{tabular}

Table 7: Geometry of relaxed icosahedral Pd (bulk nn LDF distance is $2.722 \AA$ ).

\begin{tabular}{|c|c|c|c|c|}
\hline Shell & $\mathrm{n}$ & Pd 55 & Pd 135 & $\Delta<\mathrm{nn}>(\%)$ \\
\hline \hline 0 & 1 & 0.000 & 0.000 & -4.9 \\
\hline 1 & 12 & 2.564 & 2.589 & -2.1 \\
\hline 2 & 30 & 4.470 & 4.444 & -0.59 \\
\hline 3 & 12 & 5.146 & 5.259 & -1.4 \\
\hline 4 & 20 & - & 6.415 & -0.87 \\
\hline 5 & 60 & - & 6.795 & -0.78 \\
\hline
\end{tabular}


Table 8: Geometry of relaxed fcc Ag clusters ( $\AA$ ). (Bulk LDA radius is 1.417 vs. 1.44 , experiment.)

\begin{tabular}{|c|c|c|c|c|c|}
\hline Shell & $\mathrm{n}(\mathrm{i}, \mathrm{j}, \mathrm{k})$ & $\mathrm{Ag}$ bulk & $\mathrm{Ag} 55$ & $\mathrm{Ag} 135$ & $\Delta<\mathrm{nn}>(\%)$ \\
\hline \hline 0 & $1(000)$ & 0.000 & 0.000 & 0.000 & -0.87 \\
\hline 1 & $12(110)$ & 2.834 & 2.806 & 2.810 & -0.73 \\
\hline 2 & $6(200)$ & 4.008 & 4.008 & 3.996 & -0.90 \\
\hline 3 & $24(211)$ & 4.909 & 4.849 & 4.855 & -0.80 \\
\hline 4 & $12(220)$ & 5.668 & 5.492 & 5.661 & -1.0 \\
\hline 5 & $24(310)$ & 6.337 & - & 6.322 & -1.0 \\
\hline 6 & $8(222)$ & 6.942 & - & 6.918 & -0.68 \\
\hline 7 & $48(321)$ & 7.498 & - & 7.361 & -2.1 \\
\hline
\end{tabular}

Table 9: Geometry of relaxed icosahedral Ag (bulk nn LDF distance is $2.834 \AA$ ).

\begin{tabular}{|c|c|c|c|c|}
\hline Shell & $\mathrm{n}$ & $\mathrm{Ag} 55$ & $\mathrm{Ag} 135$ & $\Delta<\mathrm{nn}>(\%)$ \\
\hline \hline 0 & 1 & 0.000 & 0.000 & -5.3 \\
\hline 1 & 12 & 2.691 & 2.683 & -2.2 \\
\hline 2 & 30 & 4.664 & 4.632 & -0.67 \\
\hline 3 & 12 & 5.348 & 5.452 & -1.3 \\
\hline 4 & 20 & - & 6.640 & -0.65 \\
\hline 5 & 60 & - & 7.091 & -0.44 \\
\hline
\end{tabular}


Table 10: Geometry of the relaxed fcc octahedra. Shell radius in $\AA$ (and average deviation from bulk $\mathrm{nn}$ spacing in \%).

\begin{tabular}{|c|c||c|c|c|}
\hline Shell & $\mathrm{n}(\mathrm{i}, \mathrm{j}, \mathrm{k})$ & $\mathrm{Ru} 140$ & $\mathrm{Pd} 140$ & $\mathrm{Ag} 140$ \\
\hline \hline 1 & $6(100)$ & $1.82(0.24)$ & $1.92(-0.54)$ & $2.00(-0.04)$ \\
\hline 2 & $8(111)$ & $3.24(-0.15)$ & $3.32(-0.54)$ & $3.48(-0.19)$ \\
\hline 3 & $24(210)$ & $4.20(-1.04)$ & $4.26(-0.56)$ & $4.46(-0.27)$ \\
\hline 4 & $6(300)$ & $5.52(-2.26)$ & $5.62(-1.12)$ & $5.92(-0.74)$ \\
\hline 5 & $24(221)$ & $5.53(-1.74)$ & $5.79(-1.20)$ & $6.03(-0.94)$ \\
\hline 6 & $24(311)$ & $6.10(-1.97)$ & $6.37(-0.92)$ & $6.65(-0.71)$ \\
\hline 7 & $24(320)$ & $6.60(-2.06)$ & $6.80(-2.26)$ & $7.12(-1.81)$ \\
\hline 8 & $24(410)$ & $7.55(-3.36)$ & $7.72(-2.73)$ & $8.07(-2.41)$ \\
\hline
\end{tabular}

Table 11: Coordination analysis for the clusters. $S=$ number of surface atoms, column numbers are coordination; bulk=12, (111) $=9,(100)=8,(110)=7$.

\begin{tabular}{|c||c|c|c|c|c|c|c|c|c|}
\hline Cluster & S/N & 12 & 11 & 9 & 8 & 7 & 6 & 5 & Ave. \\
\hline \hline icos55 & 0.76 & 13 & - & - & 30 & - & 12 & - & 8.51 \\
\hline $\mathrm{fcc} 55$ & 0.76 & 13 & - & - & 6 & 24 & - & 12 & 7.85 \\
\hline $\mathrm{i} \cos 135$ & 0.68 & 43 & 12 & 20 & - & 60 & - & - & 9.33 \\
\hline $\mathrm{fcc} 135$ & 0.68 & 43 & 12 & 8 & 24 & - & 48 & - & 8.98 \\
\hline $\mathrm{i} \cos 140$ & 0.66 & 48 & 7 & 20 & 25 & 35 & 5 & - & 9.09 \\
\hline $\mathrm{fcc} 140$ & 0.69 & 44 & - & 48 & - & 24 & 24 & - & 9.09 \\
\hline
\end{tabular}




\section{DISTRIBUTION:}

2 MS1413 D. R. Jennison, 1114

2 MS1111 P. A. Schultz, 9221

2 MS1111 M. P. Sears, 9221

1 MS1413 T. A. Michalske, 1114

2 MS0710 T. Klitsner, 4423

1 MS0710 G. A. Carlson, 6211

1 MS1436 LDRD Office, 4523

1 MS9018 Central Technical Files, 8523-2

5 MS0899 Technical Library,4414

1 MS0619 Print Media, 12615

2 MS0100 Document Processing, 7613-2 For DOE/OSTI 
